\title{
Generation of Transgenic Zebra Finches with Replication-Deficient Lentiviruses
}

\author{
Tarciso A.F. Velho ${ }^{1}$ and Carlos Lois \\ Department of Neurobiology, University of Massachusetts Medical School, Worcester, Massachusetts 01655
}

Zebra finches have been a rich experimental system for studying neurobiological questions of relevance to human health for decades. In particular, finches are the leading nonhuman model organisms for investigating the biological basis of vocal learning, a critical behavioral substrate for speech acquisition. In addition, zebra finches are an ideal system for the study of brain asymmetry, hormonal control of brain development, physiological function of sleep, sex differences in the brain, behavioral-induced gene expression, and adult neurogenesis, among other questions. Despite their importance for neurobiology, the usefulness of finches as an experimental system has been restricted by a lack of genetic manipulation methods. To overcome this barrier, our laboratory has developed methods for generating transgenic birds, including zebra finches. The successful implementation of this transgenic technology by multiple research laboratories has the potential to dramatically accelerate the progress of our understanding of the genetic basis of complex biological processes such as vocal learning. Moreover, the ability to genetically manipulate zebra finches could also be used to generate novel genetic models for human disorders that cannot be studied elsewhere or that can be more easily studied in this small bird. Here, we describe a protocol to generate transgenic zebra finches using recombinant lentiviruses.

It is essential that you consult the appropriate Material Safety Data Sheets and your institution's Environmental Health and Safety Office for proper handling of equipment and hazardous materials used in this protocol.

\section{Reagents}

Animal tattoo ink paste (Ketchum 329AA)

Dulbecco's PBS (D-PBS) modified (Hyclone SH30264.02)

Fast Green FCF (0.5\%, prepared in D-PBS and filter-sterilized)

Lentivirus containing transgene of interest

Generate lentivirus as described in Lois (2005). We use the second-generation packaging system that consists of the $p C M V$-VSVg envelope plasmid, the $p C M V$-DeltaR8.91 packaging plasmid, and a transfer plasmid of choice such as FUGW to generate transgenic animals expressing green fluorescent protein. The transfer plasmid can be easily modified to include a gene of interest or to express interference RNAs against a specific target. All plasmids used in this protocol can be obtained from a commercial plasmid depository such as Addgene. We recommend using viral titers of at least $5 \times 10^{7}$ transforming units $/ \mu \mathrm{L}$.

Mineral oil

\footnotetext{
${ }^{1}$ Correspondence: tarciso.velho@umassmed.edu

(C) 2014 Cold Spring Harbor Laboratory Press

Cite this protocol as Cold Spring Harb Protoc; doi:10.1101/pdb.prot084608
} 


\section{Equipment}

Silicone elastomer (Kwik-cast; WPI Inc.)

Sylgard 184 Elastomer Kit (Dow Corning Inc.)

Zebra finches (adult male and female breeders and fosters)

In this protocol, injected eggs are not returned to their parents because the parents will often abandon the nests and thus eggs will not hatch. Instead, foster parents are used to ensure proper incubation of the eggs. In addition, because a large percentage of manipulated eggs will not hatch due to the injection procedure, the use of fosters can help to reduce the number of breeders used.

Bird identification bands (leg bands)

Breeding cages $(\sim 40 \times 50 \times 35 \mathrm{~cm})$

Dissecting microscope (fluorescence)

DNeasy Blood \& Tissue Kit (Qiagen; optional; see Step 17)

Egg incubator (Alpha-Genesis or Grumbach; see Step 13)

Fiber optic illuminator

Fine forceps

Fine-tip marker

Flight cages $(\sim 50 \times 150 \times 60 \mathrm{~cm})$

Heparinized capillary tube (optional; see Step 17)

Hydraulic injector (e.g., Narishige MO-10)

Micromanipulator

Needles (30-gauge)

Nesting materials (coconut husk and sisal fibers)

Polymerase chain reaction (PCR) micropipettes (Drummond 1-5 $\mu \mathrm{L}$ )

Pipette beveller (e.g., Sutter BV-10)

Pipette puller

Plastic finch nest box (Lady Gouldian Finch; $12.7 \times 10.16 \times 12.7 \mathrm{~cm}$ )

Sterile cotton applicators

Sterile filter paper $\left(0.5 \mathrm{~cm}^{2}\right)$

Surgical microscope (preferably, a stereomicroscope)

Zebra finch dummy eggs

In this protocol, we inject zebra finch embryos of freshly laid eggs (unincubated) with replication-deficient lentiviruses. These embryos are then incubated in an artificial incubator and hatchlings (mosaics) are raised by foster parents. Once mosaics reach adulthood, they are crossed and their offspring screened for germline transmission of the transgene. See Fig. 1 for an overview.

\section{Preparation for Injection}

1. Pair adult wild-type male and female zebra finches in large flight cages (8 pairs/cage) to breed. Add nest boxes and nesting materials.

Once the zebra finches have been paired, it may take them 1-2 wk to start laying eggs. Three flight cages should produce at least six to eight eggs per day for 2-3 wk. Production will decline after that.

2. Establish pairs of foster parents in small breeding cages, with the goal of having at least one foster pair ready for each day of injection. Add nest boxes and fresh nesting material $4-5 \mathrm{~d}$ before embryo injection. Stagger putative foster parents by providing nest boxes and fresh nesting material to one cage per day. Collect eggs daily, keeping detailed records of laying activity, and replace them with plastic ones.

Establishing a reliable set of foster parents is crucial. Select pairs that have successfully raised healthy clutches and house them in individual cages. Foster parents will be ready to receive hatchlings $14 \mathrm{~d}$ after their first egg was laid. To keep breeding pairs healthy, give them breaks between breeding cycles, in particular after they have sat on their eggs or raised a clutch. 


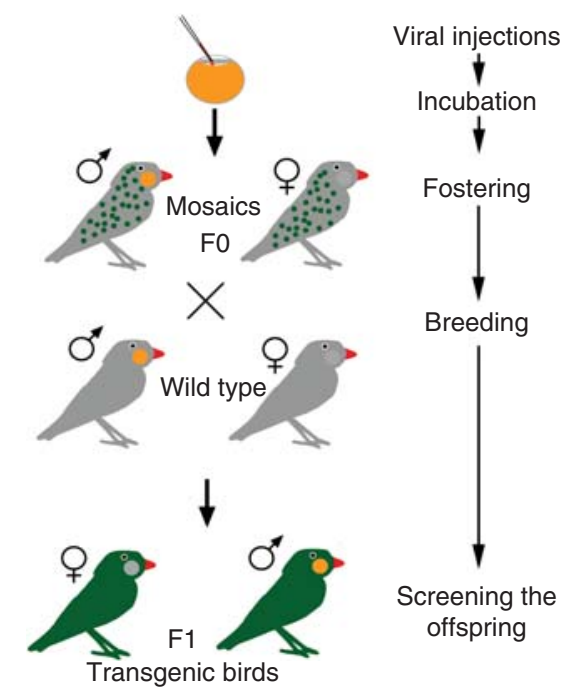

FIGURE 1. Schematic representation of lentiviral mediated transgenesis in zebra finches.

3. Make injection needles by pulling PCR micropipettes $(1-5 \mu \mathrm{L})$ with a pipette puller. With fine forceps, break the tip so the outer diameter is $\sim 15-20 \mu \mathrm{m}$, and bevel the tip at $35^{\circ}$ with a pipette beveller. Sterilize all glass pipettes in an autoclave before use.

4. Prepare egg holders by making silicone molds of the bottom halves of eggs with the Sylgard 184 Elastomer Kit. Prepare a few molds of different sizes to have a tight fit for all egg sizes and shapes.

\section{Generating Mosaics}

5. Collect eggs from the breeding pairs established in Step 1 every morning. Keep the eggs at room temperature until use.

If necessary, eggs can be kept overnight at $16^{\circ} \mathrm{C}$ before use, although this significantly reduces hatching rates.

6. On the day of injection, backfill a beveled, sterile pipette with mineral oil. Insert the plunger that comes with the PCR micropipettes and push it until the oil reaches the tip, making sure there are no air bubbles. Place the pipette into the holder of the hydraulic injector. To facilitate visualization of the pipette tip, gently apply ink to the outer tip of the pipette with a black marker and let the ink dry.

We use a Narishige hydraulic injector. Other injectors, pneumatic or hydraulic, may use a different type of capillary glass and often do not involve plungers, thus the loading procedure will be different.

7. Mix an aliquot of virus $(\sim 3 \mu \mathrm{L})$ with 10 volumes of sterile Fast Green FCF ( $0.5 \%$ in D-PBS) and load the viral particles into the injection pipette by gently applying negative pressure through the hydraulic injector.

8. Place a freshly laid egg in an egg holder and locate the embryo by shining light horizontally through the egg with the articulated arm of a fiber optic illuminator. Gently mark the embryo location with a fine-tip marker. Be careful not to reorient the embryo while moving the egg.

9. Clean the egg shell around the embryo location with a sterile cotton applicator and D-PBS to prevent contamination during the window opening process.

10. Under a surgical microscope, make a small hole (window) of 1 to $1.5 \mathrm{~mm}$ in diameter in the previously marked spot by carefully piercing a circle in the shell using a 30-gauge needle. Gently lift the shell without rupturing the shell membranes. With fine forceps, clear the window of shell debris and add a drop of sterile D-PBS to avoid air exposure of the embryo and to prevent the formation of air bubbles in the egg. Finally, remove the shell membranes with fine forceps. 
11. Locate and center the blastodisc (embryo) by tilting the egg until the blastodisc is aligned with the center of the window. Lower the loaded pipette with the micromanipulator until it touches the vitelline membrane that covers the embryo and pierce the embryo by forcefully advancing the pipette. Once you have pierced the embryo, gently retract the pipette until the tip is just below the embryo, and slowly start delivering the virus (at a rate of up to $250 \mathrm{~nL}$ in $4 \mathrm{~min}$ ) into the subgerminal space (i.e., the virtual space beneath the embryo; see Fig. 2B).

The blastodisc will be visible as a white, circular mass floating on top of the yolk. After injection, you should see viral solution spreading beneath the embryo. See Bellairs and Osmond (2005) for a more detailed description of the avian egg and early embryo anatomy.

We have successfully generated transgenic animals with multiple penetrations per embryo (by injecting 10$20 \mathrm{~nL}$ per penetration at a rate of $\sim 0.5 \mathrm{~nL} / \mathrm{sec}$ and $10-20$ penetrations for a total of $250 \mathrm{~nL}$ ). Whether to perform single or multiple injections should be determined by the experimenter according to the infection levels achieved: multiple penetrations may result in higher levels of labeling but may increase damage to the embryo and consequently decrease the hatching rates.

12. After injection(s), carefully reposition the embryo away from the window by rotating the egg axially. Remove the D-PBS using sterile filter paper until the egg's surface is dry, and seal the window using a silicone elastomer (Kwik-cast). Let the polymer set (10-15 min), and transfer the egg to the incubator.

During incubation, the air space located at the blunt end should be positioned higher than the sharp or tapered end of the egg (Fig. 2A) to ensure that the embryo's head will develop toward it. Otherwise, embryos may develop in the wrong orientation and will not be able to breathe during the piping process.

13. Incubate eggs at constant $37^{\circ} \mathrm{C}$ and $50 \%$ relative humidity. Turn the eggs hourly until they hatch $14-15$ d later.

Most small egg incubators have automated functions to control temperature, humidity, and turning conditions, but we prefer Alpha-Genesis incubators. Alternatively, we have also used Grumbach incubators, but in this case the eggs need to be transferred from the rocking platform in the last day of incubation, or the hatchlings will be crushed by the egg rocking mechanism.

14. Upon hatching, inspect the animals under a fluorescence dissecting microscope to assess somatic labeling. Hatchlings will be genetic mosaics, and a portion of their cells should carry the transgene.

Levels of labeling vary significantly; hatchlings without visible widespread somatic labeling are unlikely to show germline transmission. The percentage of germ cells (sperm or oocytes) that carry the transgene will determine the germline transmission frequency (i.e., the ratio of transgenic offspring to total offspring).

A

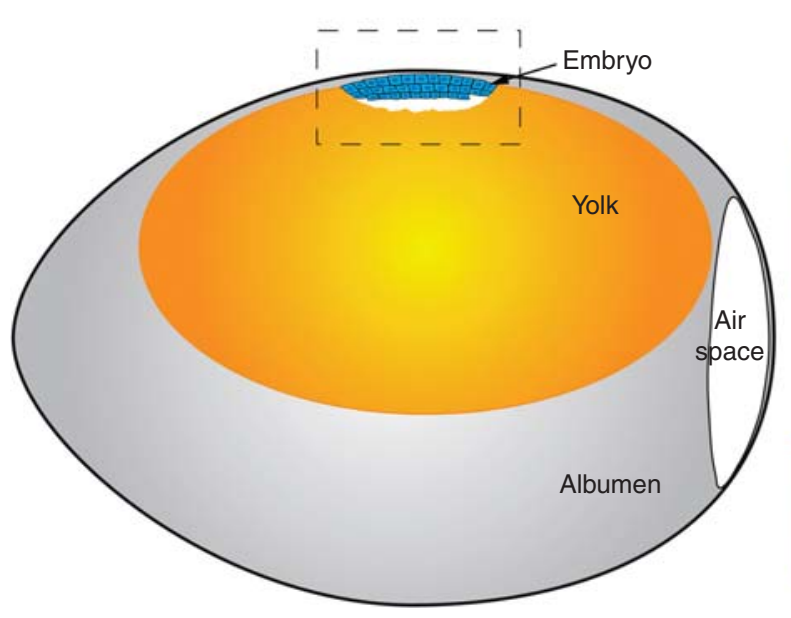

B
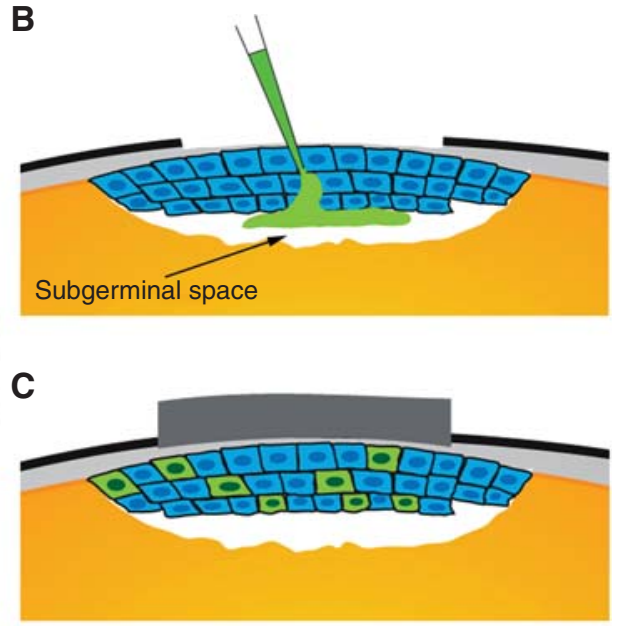

FIGURE 2. Schematic representation of viral injections into avian embryos. (A) View of a cross section of the egg depicting the early embryo; $(B)$ detailed view depicting the injection of viral solution into the embryo using a glass pipette; $(C)$ the virus will infect a portion of the embryonic cells and integrate into their genomes (green cells). 
T.A.F. Velho and C. Lois

15. Transfer labeled hatchlings to foster parents along with the egg shells.

Parents usually eat egg shells after hatching.

Transferring three to four hatchlings per nest (rather than only 1-2) increases the chance of successful fostering. Thus, use wild-type companions to reach three to four hatchlings per nest if necessary. Tattoo the wild-type companions for identification. To tattoo hatchlings, dip a 30-gauge needle in animal tattoo ink and gently pierce the skin several times, clean the ink excess, and repeat if the tattoo is not clearly visible. We prefer to tattoo along the back near the hind legs, as the skin is accessible and the tattoo is easier to see later. Band the animals as soon as they can hold a band ( $\sim 2 w k$ after hatching).

Screening Mosaic Offspring for Germline Transmission

16. At sexual maturity ( $\sim 3$ mo after hatching), breed mosaics to wild-type or other mosaic finches to generate transgenic offspring.

To increase the number of potential transgenic lines generated and minimize time spent incubating eggs, transfer the mosaics' eggs to the incubator. Hatchlings can be quickly screened (see below) and positive birds transferred to foster parents.

Although crosses of two mosaics could result in offspring carrying multiple copies of the transgene, such events should be relatively rare and the risk is outweighed by the advantages of potentially increasing the transmission rates for a given pair while reducing the number of cages and animals used. Moreover, multiple copies of the insert can be easily segregated in a few generations if needed.

17. Screen the offspring.

- For transgenic animals carrying visible markers such as fluorescent proteins, screen by inspecting the hatchlings.

- For animals that do not carry visible markers, or for animals in which the markers cannot be easily detected with a dissecting fluorescent microscope, nick the alar vein that runs along the ventral surface of the wing, draw $2-5 \mu \mathrm{L}$ of blood in a heparinized capillary tube, purify the DNA from collected blood using DNeasy Blood \& Tissue Kit (Qiagen), and genotype the animals by PCR or Southern blot.

The generation of mosaics with high levels of somatic labeling can be challenging. Two of the major bottlenecks are the ability to infect embryonic cells with lentiviruses and the ability to hatch and raise the mosaic offspring.

Embryo Labeling

Infection is highly dependent on the quality of the viral preparations. We recommend preparing multiple viral batches and testing for infectivity by injecting multiple eggs with each batch. Manipulated embryos can be killed 3-4 d postinjection and examined under the dissecting microscope to access labeling. Note that: (i) extraembryonic labeling is easily achieved and may therefore give a deceiving suggestion of successful embryo labeling, whereas labeling of the embryonic cells is significantly less efficient and (ii) labeling levels can vary dramatically, therefore we recommend examining multiple embryos per viral batch. Choose the viral batch that gives the highest level of somatic labeling.

Incubating and Hatching

Hatching rates can be affected by multiple factors. First and foremost, the quality of the reagents can have significant effects on the hatchability, especially the viral preparation. Clean viral preparations minimize embryo mortality as bacterial or yeast contamination can reduce hatching rates down to zero. Second, use sterile, aseptic injection techniques to avoid egg contamination during the injection procedure. Third, remove any eggshell debris generated during the windowing process. Finally, raising mosaic hatchlings can be a limiting factor. The health of newly hatched mosaics can be affected by the embryo manipulation process (i.e., contaminated eggs and or the damage caused by injections) and 
consequently result in rejection by the foster parents. The quality and proper timing of foster parents are also critical factors. Some pairs will be better than others at accepting and raising offspring. We recommend selecting experienced pairs that are used to the presence of experimenters. We recommend using fosters that have been sitting on their own eggs (or dummy eggs) for $14 \pm 1 \mathrm{~d}$.

\section{RELATED INFORMATION}

The use of zebra finches as an experimental system for studying neurobiological questions of relevance to human health is discussed by Zeigler and Marler (2008) and in The Zebra Finch, Taeniopygia guttata: An Avian Model for Investigating the Neurobiological Basis of Vocal Learning (Mello 2014).

\section{REFERENCES}

Bellairs R, Osmond M. 2005. The atlas of chick development. Elsevier Academic Press, London.

Lois C. 2005. Generation of transgenic animals using lentiviral vectors. In Mammalian and avian transgenesis: New approaches (eds. Pease S, Lois C) pp. 1-22. Springer, Berlin.
Mello CV. 2014. The Zebra finch, Taeniopygia guttata: An avian model for investigating the neurobiological basis of vocal learning. Cold Spring Harb Protoc doi: 10.1101/pdb.emo084574.

Zeigler HP, Marler P, ed. 2008. Neuroscience of birdsong Cambridge University Press, Cambridge. 


\section{Generation of Transgenic Zebra Finches with Replication-Deficient Lentiviruses}

Tarciso A.F. Velho and Carlos Lois

Cold Spring Harb Protoc; doi: 10.1101/pdb.prot084608 originally published online October 23, 2014

\begin{tabular}{|c|c|}
\hline $\begin{array}{r}\text { Email Alerting } \\
\text { Service }\end{array}$ & Receive free email alerts when new articles cite this article - click here. \\
\hline $\begin{array}{l}\text { Subject } \\
\text { Categories }\end{array}$ & $\begin{array}{l}\text { Browse articles on similar topics from Cold Spring Harbor Protocols. } \\
\text { Avian (39 articles) } \\
\text { DNA Delivery/Gene Transfer (344 articles) } \\
\text { DNA Delivery/Gene Transfer, general (341 articles) } \\
\text { Emerging Model Organisms (321 articles) } \\
\text { Transgenic Technology, general (187 articles) } \\
\text { Transgenics, Other Organisms ( } 34 \text { articles) } \\
\text { Use of Reporter Genes (124 articles) } \\
\text { Viral Methods ( } 113 \text { articles) }\end{array}$ \\
\hline
\end{tabular}

